Article

\title{
Development of a 3D Printed Coating Shell to Control the Drug Release of Encapsulated Immediate-Release Tablets
}

\author{
Mohammed S. Algahtani ${ }^{1, *}{ }^{\mathbb{D}}$, Abdul Aleem Mohammed ${ }^{1}$, Javed Ahmad ${ }^{1}$ and Ehab Saleh ${ }^{2} \mathbb{C}$ \\ 1 Department of Pharmaceutics, College of Pharmacy, Najran University, Najran 66433, Saudi Arabia; \\ aaleem@nu.edu.sa (A.A.M.); jaahmed@nu.edu.sa (J.A.) \\ 2 Future Manufacturing Processes Research Group, Faculty of Engineering and Physical Sciences, University \\ of Leeds, Leeds LS2 9JT, UK; E.Saleh@leeds.ac.uk \\ * Correspondence: msalqahtane@nu.edu.sa
}

Received: 23 May 2020; Accepted: 19 June 2020; Published: 22 June 2020

\begin{abstract}
The use of 3D printing techniques to control drug release has flourished in the past decade, although there is no generic solution that can be applied to the full range of drugs or solid dosage forms. The present study provides a new concept, using the 3D printing technique to print a coating system in the form of shells with various designs to control/modify drug release in immediate-release tablets. A coating system of cellulose acetate in the form of an encapsulating shell was printed through extrusion-based 3D printing technology, where an immediate-release propranolol $\mathrm{HCl}$ tablet was placed inside to achieve a sustained drug release profile. The current work investigated the influence of shell composition by using different excipients and also by exploring the impact of shell size on the drug release from the encapsulated tablet. Three-dimensional printed shells with different ratios of rate-controlling polymer (cellulose acetate) and pore-forming agent (D-mannitol) showed the ability to control the amount and the rate of propranolol $\mathrm{HCl}$ release from the encapsulated tablet model. The shell-print approach also showed that space/gap available for drug dissolution between the shell wall and the enclosed tablet significantly influenced the release of propranolol $\mathrm{HCl}$. The modified release profile of propranolol $\mathrm{HCl}$ achieved through enclosing the tablet in a 3D printed controlled-release shell followed Korsmeyer-Peppas kinetics with non-Fickian diffusion. This approach could be utilized to tailor the release profile of a Biopharmaceutics Classification System (BCS) class I drug tablet (characterized by high solubility and high permeability) to improve patient compliance and promote personalized medicine.
\end{abstract}

Keywords: 3D printing technology; solid dosage forms; controlled release; propranolol $\mathrm{HCl}$; cellulose acetate; release kinetic; personalized medicine

\section{Introduction}

The concept of a one-size-fits-all dosage form is challenging and difficult to achieve due to the high individual variability related to genetics, ethnicity, gender, age, and patient weight [1]. In real practice, some patients will be exposed to either high or low doses, with exposure to unwanted side effects. The concept of personalized medicine has grown dramatically in popularity in recent years, as dose adjustments can be made according to the pharmacokinetic and pharmacogenetic profile of the patient [2].

Three-dimensional printing technologies have been exploited for personalized drug delivery [3]. To achieve the goal of personalized medicine, the 3D printing technique was utilized to design, for example, a five-in-one dose combination polypill with defined immediate and extended-release profiles [4], a multi-layered polypill as a platform for multi-drug therapy production [5], tablets with 
fully customizable release profiles [6], and dosage forms to deliver tailored individualized precision dosing of anti-coagulant [7]. This technology can be used to fabricate pharmaceutical formulations in different sizes and shapes using a variety of materials with customized drug concentrations and release profiles that cannot be produced using conventional mass production methods in the pharmaceutical industry [8]. Different types of 3D printers using inkjet printing [9], stereolithography (SLA) [10], selective laser sintering (SLS) [11], fused deposition modeling [12], and extrusion-based pressure-assisted micro syringes [13] have been used to develop various types of drug delivery systems and novel devices such as transdermal patches [14], intrauterine and sub-cutaneous devices [15,16], and biodegradable implants [17]. Recently, Dumpa et al. developed a gastro-retentive floating pulsatile drug delivery system where the investigator enclosed a theophylline tablet prepared by direct compression into a floating shell, exploiting the 3D printing technique (hot-melt extrusion-paired fused-deposition modeling) for the treatment of chronic asthma [18].

Here, we propose a potential application of 3D printing technology to modify the release profile of an immediate-release tablet. An extrusion-based printing method was used in this work as it allows for a broad selection of suitable polymers and excipients, and also allows for printing with different polymer and excipients ratios; therefore, modified release profiles could be easily achieved by printing solid dosage forms of specific desirability, exploiting the computer-aided design (CAD) process. The current study aims to develop an encapsulating system (shell) to provide a controlled-release coating layer over an immediate-release tablet (using propranolol $\mathrm{HCl}$ (Indicardin ${ }^{\circledR}, 40 \mathrm{mg}$ tablet) as a model) to achieve a sustained release profile of the drug. Propranolol $\mathrm{HCl}$ is a beta-blocker commercially available in different dosage forms, including capsules, tablets, and oral solutions. Propranolol $\mathrm{HCl}$ has a short elimination half-life ( $3 \mathrm{~h}$ ). Therefore, frequent dosing ( $3-4$ times a day) is prescribed to achieve optimal therapeutic efficacy. The development of a drug delivery system to deliver propranolol $\mathrm{HCl}$ at a controlled rate is required to minimize the dosing frequency and reduce fluctuations in systemic drug concentrations [19]. Based on the Biopharmaceutics Classification System (BCS), propranolol $\mathrm{HCl}$ is a class I drug (high soluble and high permeable), with a dose number (Do) of 0.0025 . These characteristics of propranolol $\mathrm{HCl}$ make it an excellent candidate for the development of modified-release dosage products [20]. The tablet was placed inside a 3D printed controlled-release shell to observe the change in dissolution profile and release kinetics of propranolol $\mathrm{HCl}$.

\section{Materials and Methods}

\subsection{Materials}

Propranolol $\mathrm{HCl}$ (Indicardin ${ }^{\circledR}, 40 \mathrm{mg}$ ) was supplied by the Najran University Hospital pharmacy, Najran, Saudi Arabia. Cellulose acetate, D-mannitol, and polyethylene glycol (PEG) 6000 were purchased from Sigma-Aldrich (Gillingham, UK). Ethanol, acetone, dimethyl sulfoxide (DMSO) were purchased from UFC Biotech (Riyadh, KSA), and all other reagents were of analytical grade.

\subsection{Preparation of Material for Extrusion-Based 3D Printing of Controlled-Release Tablet Shells}

A hydrophobic polymer was selected to prepare 3D printed controlled-release shells in order to modify the release of enclosed immediate release tablet. Along with the polymer, a suitable filler (as a pore-forming agent to control the porosity) and a plasticizer (to improve shell plasticity) were added to the shell composition. All the optimized materials (hydrophobic polymer, filler, and plasticizer) in varying compositions were mixed properly and pulverized using a mortar and pestle. A solvent system was optimized to convert the selected materials into a paste of extrudable consistency. The selected materials were mixed with a fixed volume of the solvent system to transform into a homogenous paste with continuous stirring. 


\subsection{Process Optimization for 3D Printing of Controlled Release Tablet Shells}

Material for 3D printing was selected for the extrusion printing of controlled-release shells to encapsulate the tablet with an immediate release profile. Material extrusion requires optimization of various printing parameters in order to obtain stable deposition and a well-defined geometry of the controlled-release shells. These optimization parameters include consistency of the extruding paste, printing pressure, nozzle diameter (printer tip), and printing speed.

\subsection{Design of 3D Printed Controlled-Release Tablet Shells}

The geometry of controlled-release shell exploiting CAD was designed with respect to the dimensions of the selected tablet model. The design of the controlled-release shell was arranged in two parts: a shell body and a cap. The body of the controlled-release tablet shell was printed first and then the tablet was placed inside it, with subsequent printing of the cap of the shell. Different dimensions of controlled-release shells were printed to customize the drug release profile.

\subsection{Evaluation of Propranolol $\mathrm{HCl}$ Tablet}

\subsubsection{Assay/Drug Content}

An assay of the propranolol $\mathrm{HCl}$ tablet was performed as per the method described in the British Pharmacopoeia to determine the drug content. Twenty tablets were accurately weighed and crushed into a powder form. Propranolol $\mathrm{HCl}(20 \mathrm{mg})$ was dissolved in $20 \mathrm{~mL}$ of distilled water and shaken for $10 \mathrm{~min}$. Then, $50 \mathrm{~mL}$ of methanol was added to this and shaken for another $10 \mathrm{~min}$. A sufficient volume of methanol $(30 \mathrm{~mL})$ was then added to make the volume up to $100 \mathrm{~mL}$, and then filtered. Finally, $10 \mathrm{~mL}$ of this filtrate was diluted in $50 \mathrm{~mL}$ of methanol and the resulting solution was used to measure the absorbance at $290 \mathrm{~nm}$. The content of propranolol $\mathrm{HCl}$ was then calculated as described in the Pharmacopoeia [21]. The content of propranolol $\mathrm{HCl}$ tablet was found to be $39.78 \pm 1.14 \mathrm{mg}$, with a percentage content of $99.45 \%$ of the tablet dose.

\subsubsection{In Vitro Dissolution Study}

A dissolution study was carried out for the immediate-release tablet of propranolol hydrochloride using a United States Pharmacopeia (USP) type 1 dissolution apparatus in $0.1 \mathrm{~N} \mathrm{HCl}$ at $100 \mathrm{rpm}$. The 5-mL samples were withdrawn at regular time intervals $(5,10,15,20,30$, and $45 \mathrm{~min})$ to quantify the drug release. The withdrawn sample was filtered through a syringe filter and spectrophotometrically analyzed to estimate the concentration of propranolol hydrochloride in release media at the wavelength of $290 \mathrm{~nm}$ [22].

\subsection{Encapsulation of Propranolol $\mathrm{HCl}$ Tablets}

The formulated paste was placed in an extruding syringe and loaded into the 3D printer (Biobot 1). A $600-\mu \mathrm{m}$ nozzle tip was fixed to the extruding syringe. The printing pressure was set between 40 and 60 psi based on the paste composition and consistency. The G-code files were uploaded to the printer software and the files were run to conduct the printing process. Firstly, the body of the controlled-release shell was printed; then, the propranolol $\mathrm{HCl}$ tablet was placed inside the printed shell. The cap of the controlled-release shell was then printed over the top of the body to close the controlled-release shell. The total printing time was approximately 4-6 min for each print. The printing speed was $4 \mathrm{~mm} / \mathrm{s}$ and was kept constant. After printing, the 3D printed tablets were placed in a vacuum dryer overnight to complete the drying process. 


\subsection{Characterization of Printed Controlled-Release Shell}

\subsubsection{Fourier-Transform Infrared Spectroscopy (FTIR)}

FTIR was performed to detect drug-excipient interactions during the 3D printing process. Infrared spectra were obtained for the physical mixture of ingredients and the 3D printed controlled-release shell using an FTIR spectrometer (Agilent Cary630 FTIR). A small amount of powdered excipient mixture used in the shell preparation was analyzed at wavenumbers between 400 and $4000 \mathrm{~cm}^{-1}$. The results from the excipient physical mixture were compared to a sample of the 3D printed controlled-release shell to assess any drug-excipient interactions during the 3D printing process [23].

\subsubsection{Differential Scanning Calorimetry (DSC)}

DSC analysis was carried out for the physical mixture of excipients (cellulose acetate, D-mannitol, and PEG 6000) and the 3D printed controlled-release shell using a TA DSC25 Instrument. The physical mixture of the material and the 3D printed controlled-release shell were powdered and sieved. An accurately weighed amount of sample $(5 \mathrm{mg})$ was placed in a Tzero aluminium pan and sealed completely. The nitrogen flow rate used for the analysis was $50 \mathrm{~mL} / \mathrm{min}$ and the heating rate was set at $10^{\circ} \mathrm{C} /$ min using an empty Tzero aluminium pan as a reference [24].

\subsection{In-Vitro Dissolution Study}

\subsubsection{Drug Release Profile}

In vitro drug dissolution studies were performed for the 3D printed tablets of different compositions and sizes using USP type 1 dissolution apparatus as per the monograph for propranolol $\mathrm{HCl}$ at $100 \mathrm{rpm}$ using $900 \mathrm{~mL}$ of phosphate buffer ( $\mathrm{pH}$ 6.8) as the dissolution medium at a temperature of $37 \pm 0.5^{\circ} \mathrm{C}$. The amount of sample withdrawn was $5 \mathrm{~mL}$ at the time points of 5, 10, 15, 30, 60, 120, 240,360, 480, 600 , and $720 \mathrm{~min}$. The withdrawn sample was filtered through a syringe filter and analyzed using a UV spectrophotometer at $290 \mathrm{~nm}$. The dissolution studies were performed in triplicate and the cumulative percentage of drug release with respect to time was determined [25,26].

\subsubsection{Drug Release Kinetics}

The drug release kinetics which showed the mechanism of overall release of drug from the developed formulations were subjected to quantitative and qualitative changes in formulation designs considered as rational to understand the release mechanism. The propranolol $\mathrm{HCl}$ release kinetics from the encapsulated tablet in the printed shells of different composition and sizes were determined by using the best-fit model [27].

\section{Results and Discussion}

\subsection{Preparation of Material for 3D Printing of Controlled-Release Tablet Shells}

Pharmaceutical product development exploiting the 3D printing technique requires specific consideration of material choice in accordance with the method used for printing. For example, the fused deposition modeling (FDM) method requires the use of thermoplastic materials extruded at high temperatures. The current investigation involved the use of a pressure-assisted microsyringe (PAM) that requires materials to be extruded at room temperature in a semi-solid state, which consequently dry after extrusion from the printer.

For the shell composition, different hydrophobic polymers were investigated in order to modify the drug release of the tablet enclosed inside the core of the shell. The material was optimized for extrusion through the 3D printer and explored for its ability to build a layer-by-layer design of controlled/sustained-release shells. The selection of the polymers and all other excipients/chemicals exploited in this investigation was based on well-reported use in literature for the preparation of tablet 
shells. These excipients have been well exploited using the PAM-based 3D printing technique for pharmaceutical product development $[4,24,28,29]$ and/or the formulation of controlled/sustained-release tablets [30,31]. The polymers investigated to assess extrusion ability through the 3D printer included ethyl cellulose, hydroxyl propyl methylcellulose (HPMC), polyvinylpyrrolidone (PVP), and cellulose acetate. These polymers were dissolved in different types of solvent and tested to form a paste of desirable consistency that should be suitable for 3D printing. The solvents investigated for the formation of paste with good extrusion consistency included methanol, ethanol, isopropyl alcohol, dichloromethane, dimethyl sulfoxide, and acetone. The optimization of the solvents exploited in the preparation of a paste with good extrusion consistency should take into consideration the rheology of the formulated paste and the time required to evaporate the solvent. Solvents should not be highly evaporative, as this may cause fast drying of the extrusion material at the tip of the nozzle, ultimately causing a blockage. However, slow evaporation of the solvent may increase the time required for solidification of the printed layers and ultimately hamper the formation of the layer-by-layer 3D structure. The extrusion paste should be smooth and homogeneous in order to exhibit good flow characteristics through the nozzle tip and be free from the occurrence of large particles that may cause blockage of the nozzle tip during the extrusion process. Among the various polymers assessed for the preparation of pastes, ethylcellulose, HPMC, and PVP exhibited variable characteristics in terms of the formed paste, including consistency, rheology, and extrusion behaviour. During the deposition observation, these materials showed high wettability at the interface with the borosilicate glass substrate, which prevented the building of a layer-by-layer 3D structure. Based on the physical properties and rheological behaviour during extrusion, cellulose acetate was found to be a suitable polymer to print the controlled-release tablet shells. Polyethylene glycol (PEG) is one of the most efficient plasticizers used in polymer-based pharmaceutical product development as it has a broad range of molecular weight grades available, is nontoxic in nature, and shows miscibility with a wide range of pharmaceutical excipients as well as biodegradability [32].

Furthermore, among the different grades of PEG, the higher molecular weight grade provides a better plasticizing effect [33]; therefore, PEG 6000 was added as a plasticizer to the shell composition. The amount of solvent plays an essential role in affecting the flow behaviour of the paste. A combination of solvents was used to prepare the cellulose acetate paste for extrusion-based 3D printing. The use of a highly evaporative solvent will lead to rapid drying of the paste causing a nozzle blockage, and the use of a slowly evaporating solvent will increase the ink spreading upon printing and complicate the formation of well-defined layer-by-layer 3D structures. To overcome this, a combination of solvent mixture was used to provide the optimum rate of solvent evaporation from the paste after extrusion through the 3D printer. The miscibility/solubility profile of cellulose acetate in different solvents and the resulting solution behaviour, along with the extrudability characteristics of the paste through the 3D printer, are shown in Table 1.

The cellulose acetate was dissolved in a combination of acetone, ethanol, and DMSO in the ratio of 2.5:2.5:1 and optimized as extrusion material for the printing of controlled-release tablet shells. The DMSO added as co-solvent for cellulose acetate was within the permissible limits and concentrations safe for use in humans $[34,35]$. The optimized materials for the shell formation were composed of cellulose acetate, D-mannitol, and PEG 6000. It was mixed properly and pulverized using a mortar and pestle. Then, $3.0 \mathrm{~g}$ of the blended powder mixture of cellulose acetate, D-mannitol, and PEG 6000 was taken and mixed with a fixed volume $(2.4 \mathrm{~mL})$ of the solvent system (acetone, ethanol, and DMSO in the ratio of 2.5:2.5:1 v/v) to make a homogenous paste of a semi-solid consistency with continuous stirring. 
Table 1. Miscibility/solubility profile of cellulose acetate in different solvents and the resulting solution behaviour, along with extrudability characteristics of the paste through the 3D printer.

\begin{tabular}{|c|c|c|}
\hline Solvent & Solubility & $\begin{array}{c}\text { Solution Behavior and Extrudability } \\
\text { Through the 3D Printer }\end{array}$ \\
\hline Water & Insoluble & Insoluble mass \\
\hline Water with surfactant (Tween 80) & Insoluble & Granular mass \\
\hline Isopropyl alcohol & Insoluble & Insoluble mass \\
\hline Ethanol & Insoluble & Insoluble mass \\
\hline Dichloromethane & Insoluble & Insoluble mass \\
\hline Ethyl acetate & Partially soluble & Segregated mass \\
\hline Acetone & Soluble & $\begin{array}{l}\text { Paste extruded rapidly, but tip blockage } \\
\text { occurred due to drying of the paste over time }\end{array}$ \\
\hline Acetone/water (1:1 ratio) & Insoluble & Segregated mass \\
\hline Acetone/ethanol (1:1 ratio) & Insoluble & Gummy mass \\
\hline Acetone/isopropyl alcohol (1:1 ratio) & Insoluble & Gummy mass \\
\hline Acetone/propylene glycol (4:1 ratio) & Soluble & Paste but hindered extrusion \\
\hline Acetone/isopropyl alcohol (3:2 ratio) & Soluble & Paste but no continuous extrusion \\
\hline $\begin{array}{l}\text { Acetone/ethanol/ dimethyl sulfoxide } \\
\text { (DMSO) (2.5:2.5:1 ratio) }\end{array}$ & Soluble & $\begin{array}{l}\text { Good uniformity and continuously } \\
\text { extrudable paste }\end{array}$ \\
\hline
\end{tabular}

\subsection{Process Optimization for 3D Printing Controlled-Release Tablet Shells}

Among the various 3D printing technologies exploited for pharmaceutical manufacturing, the extrusion-based 3D printing technique was investigated for printing controlled-release shells of the encapsulated tablet. The printer explored for the study was a pressure-assisted semi-solid extrusion-based $3 \mathrm{D}$ printer (BIOBOT 1). This technique was optimized for various process parameters (such as paste consistency, nozzle diameter, printing pressure, and speed) in order to print the controlled-release shell of various configurations. Firstly, the paste was formed, which was well extruded through the printer nozzle. The cellulose acetate was dissolved in a combination of acetone, ethanol, and DMSO in a ratio of 2.5:2.5:1, along with a suitable filler (D-mannitol) and plasticizer (PEG 6000) in order to print a stable layer of controlled-release shell wall with the optimum hardness and drying time. The prepared paste was placed in an extrusion syringe with nozzles of different diameters attached to the syringe for printing. The nozzle diameter was optimized for printing. The selection of nozzle size was based on the consistency, flowability, and particle size of the paste. Tapered nozzles with inner tip diameters of 400-600 $\mu \mathrm{m}$ were optimized for printing the controlled-release shells. The printing pressure and printing speed were optimized based on the consistency of the paste. The printing pressure influences the flow behaviour of the paste through the nozzle and relies on the composition of the paste. The printing pressure was varied based on the amount of filler added to the composition of extruding paste; 40-60 psi was found to be the optimum pressure for the printing of controlled-release shells. Similarly, the printing speed of $4 \mathrm{~mm} / \mathrm{s}$ was found to be optimum for printing controlled-release shells. All the printing parameters were kept constant throughout the printing process; the optimization results are summarized in Table 2.

\subsection{Design of 3D Printed Controlled-Release Tablet Shells}

The empty shell shape was designed to be round, similar to the shape of the tablet chosen for the study. The size of the selected tablet was $8 \mathrm{~mm}$ in diameter and $2.6 \mathrm{~mm}$ in thickness. Controlled release shells of three different sizes (i.e., fixed, medium, and large) were designed to demonstrate the influence of the gap/space between the shell wall and the enclosed tablet on the dissolution time and drug release behaviour. The optimized design for printing the tablet shells was developed as a stereolithography (STL) file and ultimately sliced through a Repetier-Host to create G-code to print the controlled-release shell for the tablet (illustrated in Figure 1a). 
Table 2. Optimization for the extrusion-based 3D printing process for printing controlled-release tablet shells.

\begin{tabular}{|c|c|c|}
\hline Process Parameters & Value & Outcome/Observation \\
\hline \multirow{3}{*}{ Nozzle size $(\mu \mathrm{m})$} & $200-400 \mu \mathrm{m}$ & No extrusion \\
\hline & $400-600 \mu \mathrm{m}$ & Good extrusion with an optimum layer thickness \\
\hline & $600-800 \mu \mathrm{m}$ & Very rapid extrusion with an increased layer thickness \\
\hline \multirow{3}{*}{ Extrusion pressure (psi) } & $20-40$ psi & No extrusion \\
\hline & $40-60$ psi & Good extrusion with an optimum layer thickness \\
\hline & $60-80$ psi & Very rapid extrusion with an increased layer thickness \\
\hline \multirow{3}{*}{ Printing speed $(\mathrm{mm} / \mathrm{s})$} & $2 \mathrm{~mm} / \mathrm{s}$ & Slow printing \\
\hline & $4 \mathrm{~mm} / \mathrm{s}$ & Optimum printing \\
\hline & $6 \mathrm{~mm} / \mathrm{s}$ & Rapid printing, sagging due to wetting \\
\hline \multirow{2}{*}{ Nozzle shape } & Blunt tip & Tip blockage and hindered extrusion \\
\hline & Tapered tip & Smooth and continuous extrusion \\
\hline
\end{tabular}

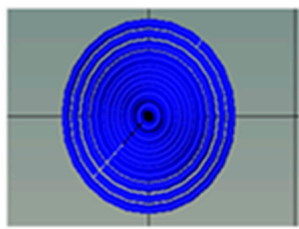

Base Spiral

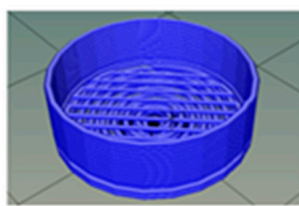

Shell body

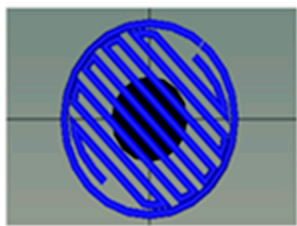

Base mesh

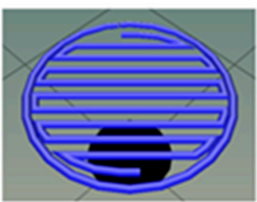

cap mesh

(a)

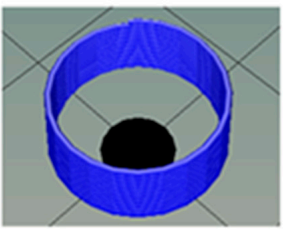

shell wall

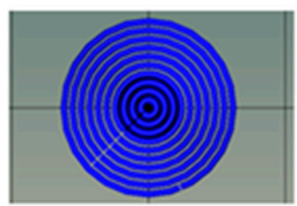

cap spiral

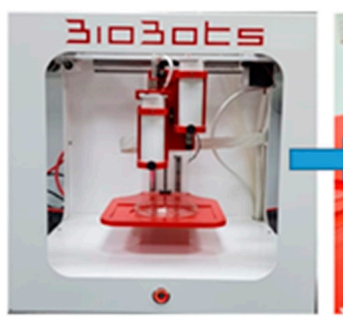

3D PRINTER (Biobot 1)
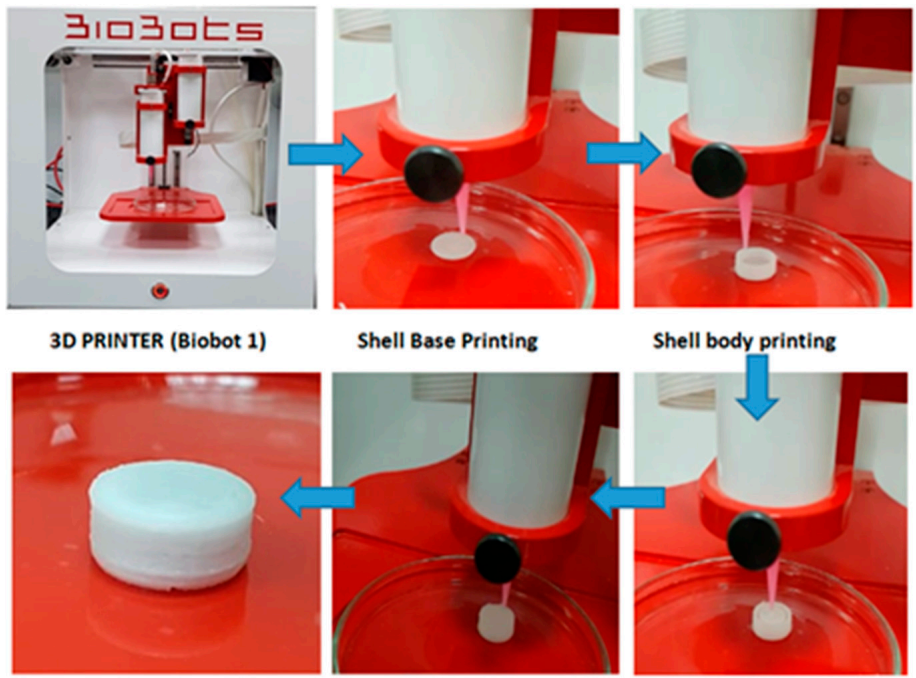

Shell Base Printing

Shell body printing

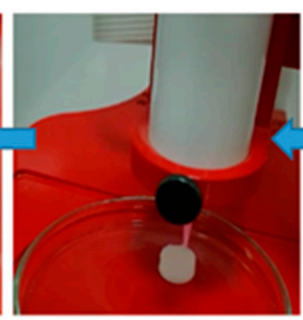

Cap printing

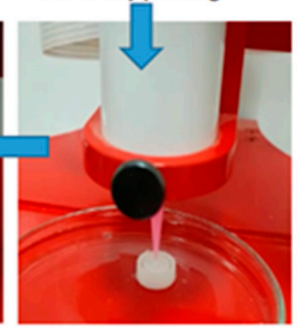

30 Printed Tablet

(b)

Figure 1. (a) Stereolithography (STL) files illustrating the design of shell body/wall and cap. (b) The printing process is enclosing the marketed tablet inside the 3D printed controlled/sustained-release shell. 


\subsection{Printing of Controlled-Release Shells to Enclose Propranolol Hcl Tablets}

After the optimization of process parameters for 3D printing of the controlled-release shell, cellulose acetate along with D-mannitol and PEG 6000 were converted into a paste by dissolving them in a combination of acetone, ethanol, and DMSO at a ratio of 2.5:2.5:1. The paste was placed into the extruding syringe immediately to avoid unnecessary loss of solvent that could cause drying of paste and influence its consistency. During the filling of paste into extruding syringe, care should be taken to avoid air bubble entrapment as its presence may influence the relaxation behavior of the paste. The air bubbles were minimized by transferring the paste from an initially filled syringe to another syringe with a seal placed near the barrel tip by using a syringe connector. The printing was done on a glass substrate. Once the body of the shell was printed, the marketed tablet was placed inside the shell with the use of tweezers, and then the cap was printed over the top of the enclosed tablet. The design geometry of the cap and base was identical to provide a uniform and controlled/sustained release of propranolol $\mathrm{HCl}$ from the developed formulation system. The first layer of the cap design was a mesh pattern to help the tablet remain intact in the center of the shell, whereas the second layer of the cap was printed over the first layer in a spiral pattern (illustrated in Figure 1b). This printed formulation system enclosing the tablet was then tested to provide a proof of concept for the 3D printing technique used to modify the drug release profile of an immediate-release tablet.

\subsection{Characterization of 3D Printed Controlled-Release Shells Enclosing the Immediate-Release Tablets}

FTIR and DSC analysis were performed in order to determine the potential impact of various unit operations such as grinding, mixing, solvent addition, paste formation, and drying involved during the 3D printing process on the material's behaviour. Furthermore, possible interactions between the excipients during formulation development were also assessed. The DSC thermogram of the physical mixture containing PEG 6000, D-mannitol, and cellulose acetate exhibited endothermal peaks (melting point) at $61.22{ }^{\circ} \mathrm{C}, 168.94{ }^{\circ} \mathrm{C}$, and $321.13^{\circ} \mathrm{C}$, respectively, while the $3 \mathrm{D}$ printed shell showed no significant changes in the endothermic peaks compared to the excipients used in its formulation (Figure 2a). This reveals that there were no significant interactions between formulation excipients during the printing process. Similarly, the FTIR spectra of the physical mixture of the materials (PEG 6000, D-mannitol, and cellulose acetate) and the 3D printed shell did not exhibit any significant shift in the position of the characteristic peak (Figure 2b). The FTIR and DSC results indicate that there were no significant interactions between formulation excipients during the $3 \mathrm{D}$ printing process.

\subsection{In Vitro Dissolution Study}

\subsubsection{Influence of the Composition of 3D Printed Controlled-Release Shells}

Before performing the dissolution studies of the enclosed tablet, the sealing of the shell was investigated. The tablet model was soaked with color dye (methylene blue) and enclosed inside the 3D printed controlled-release shell. Then, the enclosed shell was placed in a 100-mL beaker filled with distilled water. The visual observation of the gradual change of the color intensity of the water by the methylene blue at a different time intervals (Figure 3) proves that there was no sudden leakage of the tablet content from the shell, and that the release of methylene blue through the shell was controlled.

The in vitro dissolution study was carried out to determine the release of propranolol $\mathrm{HCl}$ from the developed 3D printed shell and the encapsulated tablet. The dissolution study of the tablet model revealed an immediate release profile of propranolol $\mathrm{HCl}$, with $>95 \%$ of the drug released within $20 \mathrm{~min}$. It was found that the release of propranolol $\mathrm{HCl}$ from the 3D printed shell showed controlled/sustained release compared to the tablet model (Figure 4a). This result validates the proof of concept that the 3D printing technique has potential applications in modifying the drug release profile of conventional immediate-release tablets.

The composition of the controlled-release shells (shells A, B, and C), as shown in Table 3, influenced the release profile of propranolol $\mathrm{HCl}$ (Figure 4a). Among the three different shell formulations, shell $\mathrm{A}$ 
exhibited $84 \%$ propranolol release while shell B and C allowed for $66 \%$ and $48 \%$ release of propranolol $\mathrm{HCl}$ after $12 \mathrm{~h}$, respectively. Figure $4 \mathrm{~b}$ showed the intact shell after the 12-h dissolution time, and the cross-section of the shell system showed the expansion of the disintegrated tablet inside the shell without breaking it during the dissolution.

The alteration in release profiles of propranolol $\mathrm{HCl}$ was due to the variability in the amount of rate-controlling polymer (cellulose acetate) and the pore-forming agent (D-mannitol) used in the formulation composition. The results demonstrate that an increase in the amount of D-mannitol in the shell composition led to an increase in drug release. This behaviour is correlated to the micropores formed by the rapid dissolution of D-mannitol in the controlled-release shell, which ultimately enhances the water intake, driven by the difference in the osmotic pressure across the shell. This facilitates faster dissolution of the enclosed tablet compared to the shell composition with less D-mannitol. Khaled et al. also reported the impact of variable amounts of cellulose acetate as a drug release retardant in their investigations $[4,28]$.

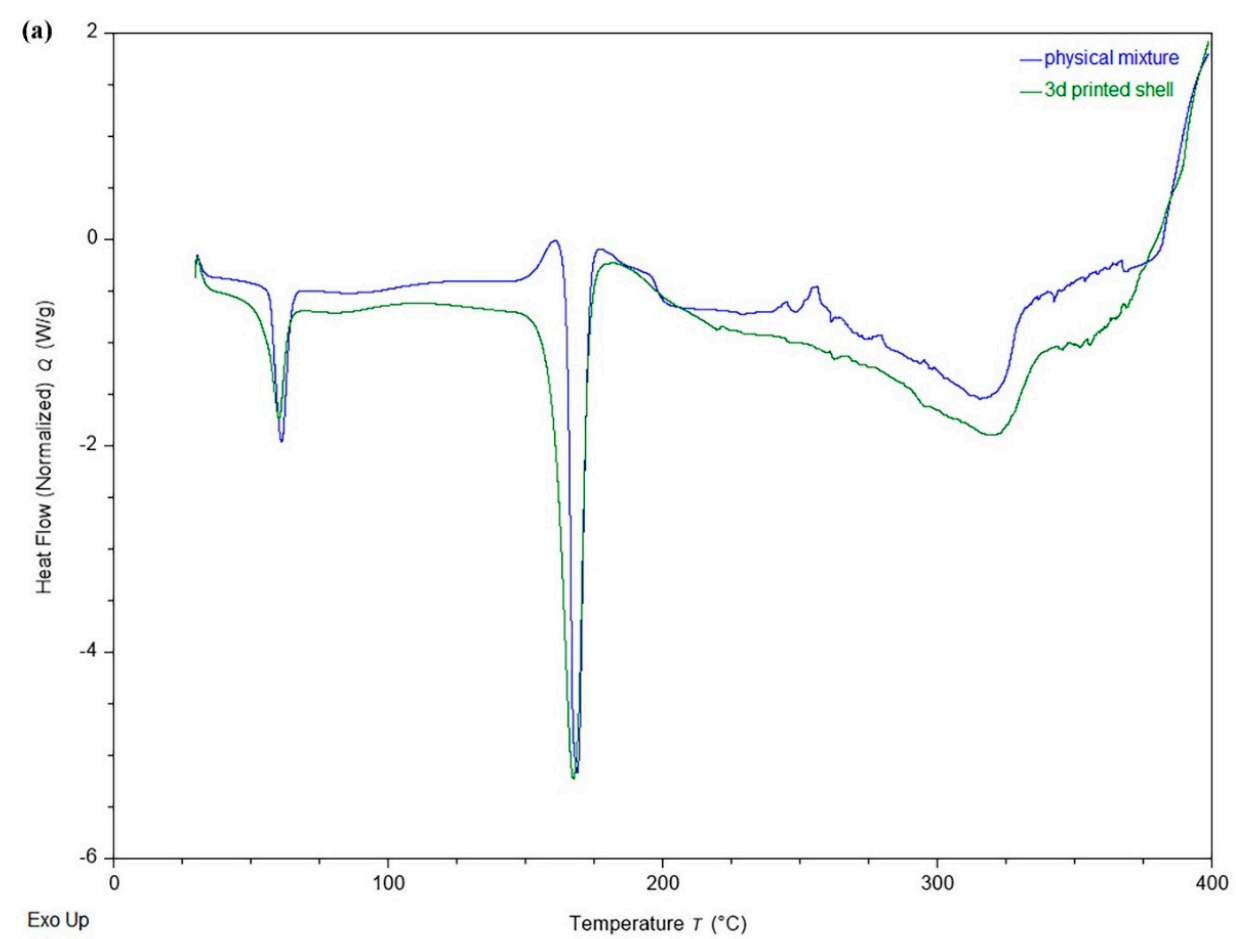

(b)

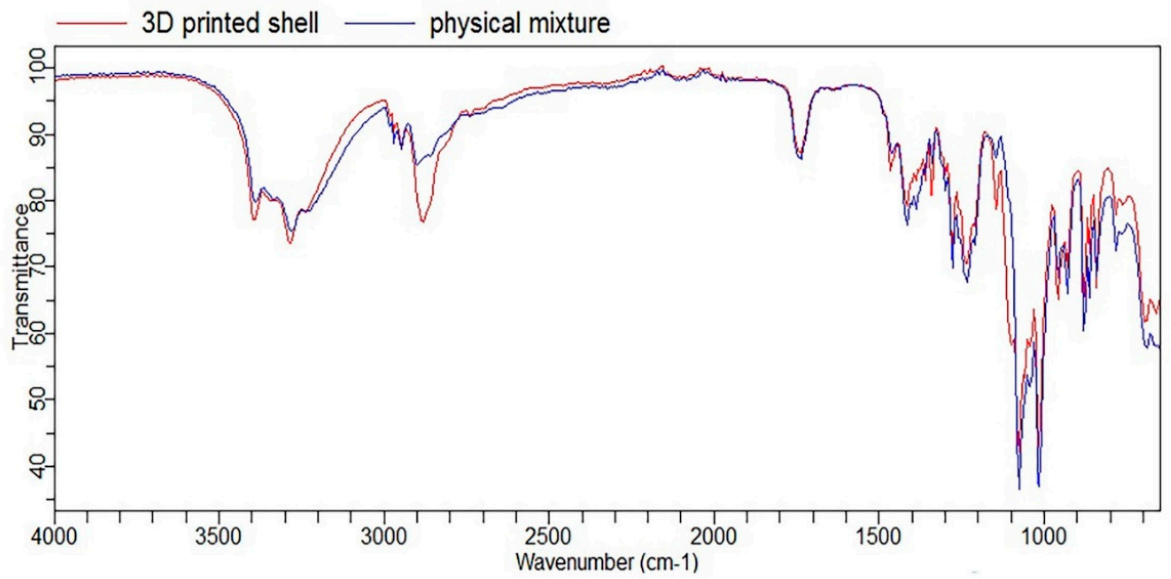

Figure 2. (a) Differential scanning calorimetry (DSC) thermogram of a physical mixture of excipients and the 3D printed shell; (b) Fourier-transform infrared spectroscopy (FTIR) spectra of a physical mixture of excipients and the 3D printed shell. 


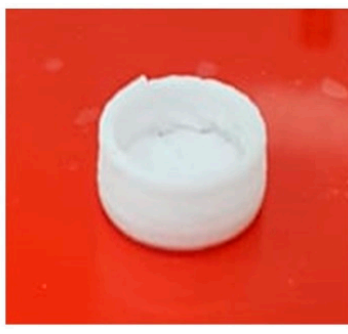

Empty shell

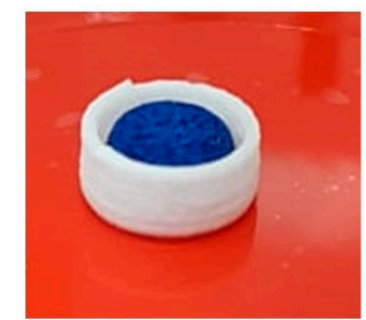

Shell containing tablet with dye

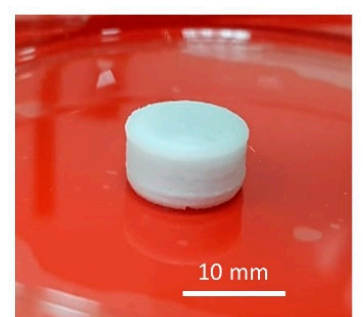

Shell enclosed with cap

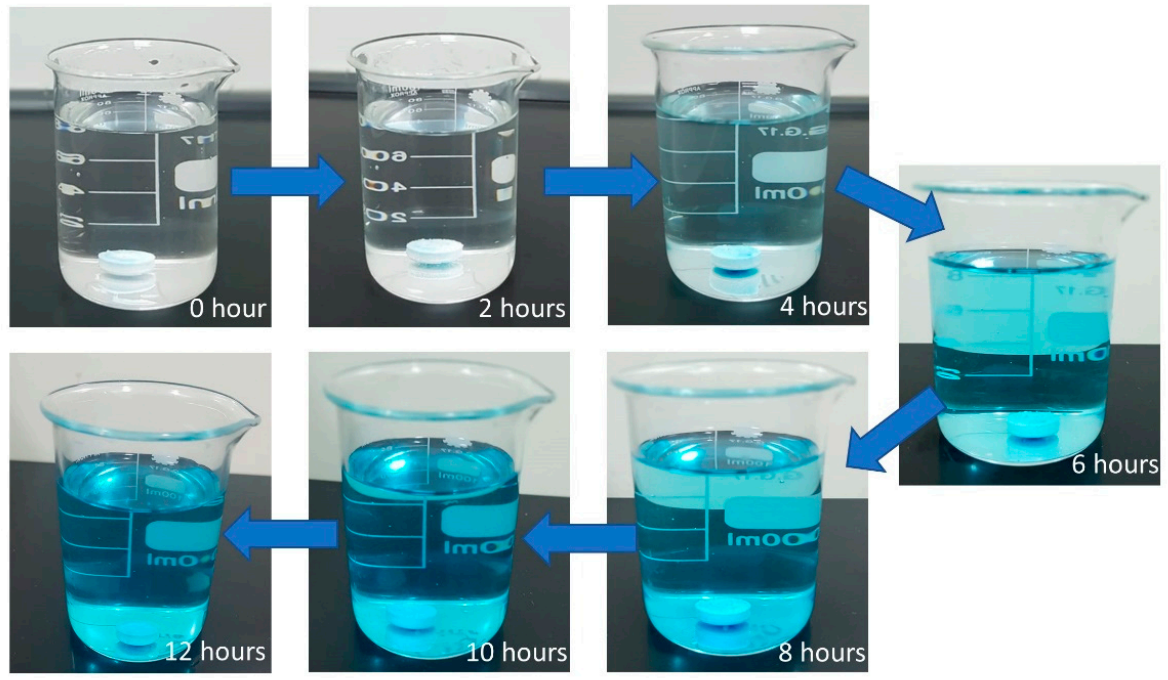

Figure 3. Illustration representing encapsulation of the propranolol $\mathrm{HCl}$ tablet soaked with color dye (methylene blue) kept inside 3D printed controlled-release shell, with the gradual increase in the intensity of the color solution showing the visual observation of methylene blue release through the 3D printed controlled-release shell at different time intervals.

Table 3. Influence of different compositions of 3D printed controlled-release shell on \% cumulative drug release after $12 \mathrm{~h}$ with phosphate buffer $\left(\mathrm{pH}\right.$ 6.8) as dissolution media at a temperature of $37 \pm 0.5^{\circ} \mathrm{C}$.

\begin{tabular}{ccccc}
\hline Formulation & \multicolumn{3}{c}{$\begin{array}{c}\text { \% Composition } \\
\text { Cellulose Acetate D-Mannitol PEG 6000 }\end{array}$} & $\begin{array}{c}\text { \% Cumulative Drug } \\
\text { Release After 12 h }\end{array}$ \\
\hline Shell A & 20 & 65 & 15 & $84.64 \pm 1.99$ \\
Shell B & 40 & 45 & 15 & $66.60 \pm 1.13$ \\
Shell C & 60 & 25 & 15 & $48.37 \pm 1.46$ \\
\hline
\end{tabular}

\subsubsection{Influence of the Size of the 3D Printed Controlled-Release Shell}

The formulation of shell B had a balanced ratio between cellulose acetate and D-mannitol (Table 3). Therefore, it was selected to assess further the influence of different sizes of 3D printed coating shells on the cumulative release of the propranolol $\mathrm{HCl}$. Three different-sized shells were printed (fixed, medium, and large). The outer and inner diameter and the gap between the shell wall and the tablet are shown in Table 4. The uncapped shell and the enclosed tablet of the three shell sizes are shown in Figure 5a. The in vitro dissolution work was carried out to evaluate the propranolol $\mathrm{HCl}$ release from the enclosed tablet inside the three different shells varying in sizes (Figure $5 \mathrm{~b}$ ). The propranolol $\mathrm{HCl}$ release rate was enhanced with respect to an increase in the size of the shell (Figure $5 \mathrm{~b}$ ).

Over a period of $12 \mathrm{~h}, 66.60 \%, 72.68 \%$, and $76.81 \%$ of propranolol $\mathrm{HCl}$ was released from the tablet enclosed in the fixed-, medium-, and large-sized shells, respectively (Table 4). As the space between the shell wall and the tablet increased, so did the amount of dissolution medium that filled that space, and consequently, there was more dissolved propranolol $\mathrm{HCl}$ from the enclosed tablet. The increase in the shell size provided a larger area (space/gap between the shell wall and the enclosed tablet) 
available for the tablet to expand during the disintegration process, resulting in more dissolution and drug release (Figure $5 \mathrm{a}, \mathrm{b}$ ). The concept of accommodating the space/gap between the enclosed tablet and the shell wall demonstrated a novel idea, paving the way to explore a different approach and hypothesis. The provided space/gap could be utilized for filling with release enhancers/solubilizers or release retardant, and the drug release from the enclosed tablet might be customized as per desirability into a delayed-release or time-dependent drug release system.

(a)

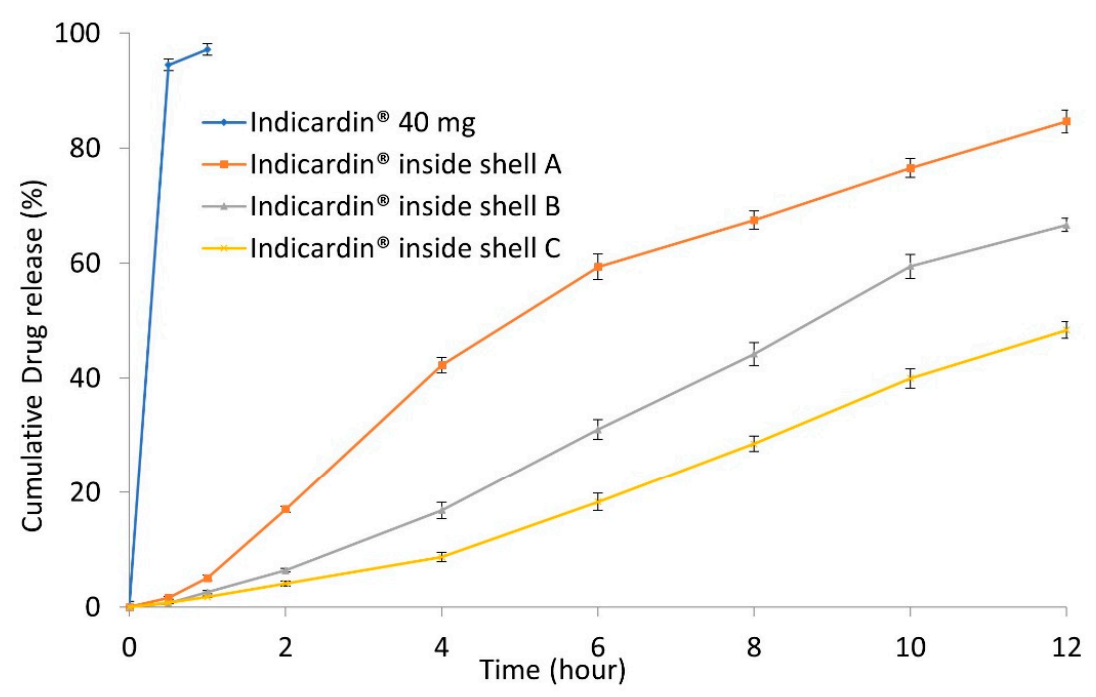

(b)

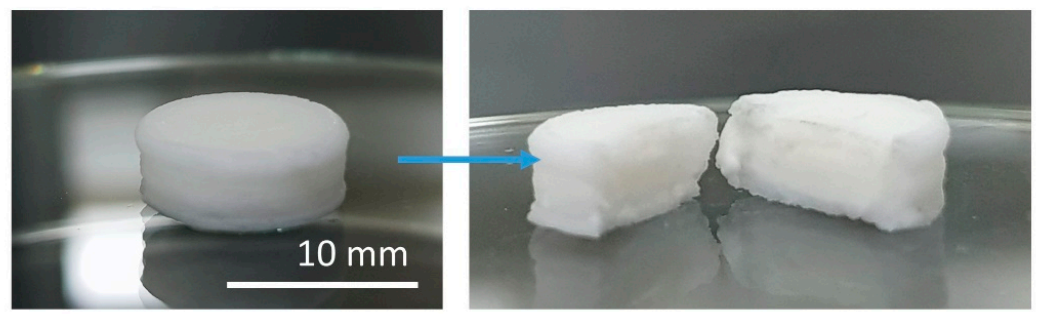

Figure 4. (a) Cumulative release of propranolol $\mathrm{HCl}$ from the model tablet with phosphate buffer (pH 6.8) as dissolution media at temperature $37 \pm 0.5{ }^{\circ} \mathrm{C}$ versus when enclosed inside 3D printed coating shells with different excipient ratios (Table 3 ). (b) The coating shell system still intact after the dissolution experiment. The cross-section of the shell shows the expansion of the propranolol $\mathrm{HCl}$ tablet inside the shell.

Table 4. Influence of different sizes of 3D printed controlled-release shells on cumulative drug release after $12 \mathrm{~h}$ with phosphate buffer ( $\mathrm{pH}$ 6.8) as dissolution media at temperature $37 \pm 0.5^{\circ} \mathrm{C}$.

\begin{tabular}{ccccc}
\hline \multirow{2}{*}{ Shell Size } & \multicolumn{3}{c}{ Shell Dimensions } & \multirow{2}{*}{$\begin{array}{c}\text { \% Cumulative Drug } \\
\text { Release After 12 h }\end{array}$} \\
\cline { 2 - 4 } & $\begin{array}{c}\text { Shell Outer } \\
\text { Diameter }\end{array}$ & $\begin{array}{c}\text { Shell Inner } \\
\text { Diameter }\end{array}$ & $\begin{array}{c}\text { The Gap between the } \\
\text { Shell Wall and Tablet }\end{array}$ & \\
\hline Fixed-sized shell & $12 \mathrm{~mm}$ & $9.6 \mathrm{~mm}$ & $0.8 \mathrm{~mm}$ & $66.60 \pm 1.13$ \\
\hline Medium-sized shell & $13 \mathrm{~mm}$ & $10.6 \mathrm{~mm}$ & $1.3 \mathrm{~mm}$ & $72.68 \pm 2.34$ \\
\hline Large-sized shell & $14 \mathrm{~mm}$ & $11.6 \mathrm{~mm}$ & $1.8 \mathrm{~mm}$ & $76.81 \pm 1.60$ \\
\hline
\end{tabular}

\subsubsection{Drug Release Kinetics}

Kinetic modeling plays an essential role in determining the drug release mechanism [36,37]. The drug release data were fitted by using the zero-order, first order, Higuchi, and Korsmeyer-Peppas models to investigate the best-fit model $[36,38]$. The results are shown in Table 5. 
(a)

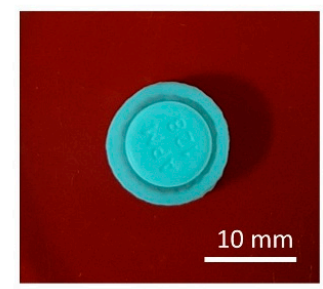

Fixed size shell

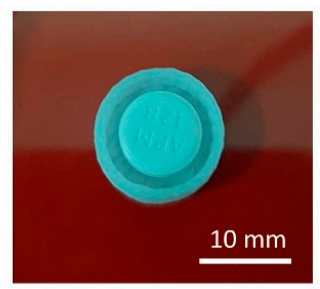

Medium size shell

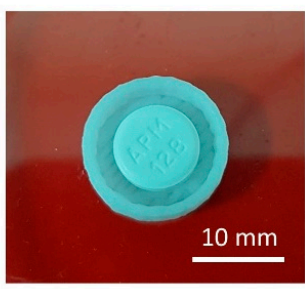

Large size shell

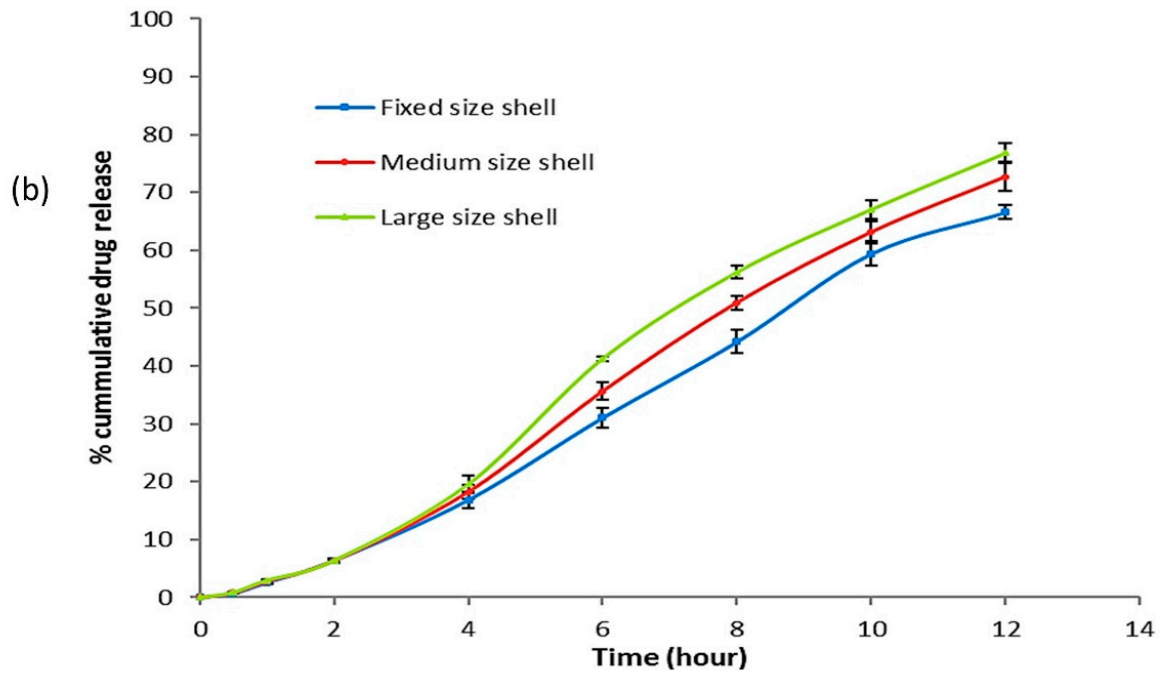

Figure 5. (a) Different sizes of 3D printed controlled-release shells illustrating the gap/space between the enclosed tablet and the shell wall. (b) Cumulative release of propranolol $\mathrm{HCl}$ with phosphate buffer ( $\mathrm{pH}$ 6.8) as dissolution media at temperature $37 \pm 0.5^{\circ} \mathrm{C}$ from $3 \mathrm{D}$ printed controlled-release shells of different sizes.

Table 5. In vitro release kinetics of propranolol $\mathrm{HCl}$ from 3D printed controlled-release shells of variable composition and size fitted to the best-fit release kinetic model.

\begin{tabular}{cccccc}
\hline \multirow{2}{*}{ Formulation Type } & Zero Order $\left(\mathbf{r}^{2}\right)$ & First Order $\left(\mathbf{r}^{2}\right)$ & Higuchi $\left(\mathbf{r}^{2}\right)$ & \multicolumn{2}{c}{ Korsmeyer-Peppas } \\
\cline { 5 - 6 } & & & & & $n$ Value \\
\hline Composition-based & & & & \\
\hline Formulation A & 0.9521 & 0.9939 & 0.9906 & 0.9662 & 0.542 \\
Formulation B & 0.9937 & 0.9741 & 0.9503 & 0.9954 & 0.619 \\
Formulation C & 0.9817 & 0.9541 & 0.9106 & 0.9959 & 0.576 \\
\hline Size-based & & & & & \\
\hline Fixed-sized shell & 0.9937 & 0.9741 & 0.9503 & 0.9954 & 0.619 \\
Medium-sized shell & 0.9949 & 0.9622 & 0.9516 & 0.9964 & 0.621 \\
Large-sized shell & 0.9902 & 0.9583 & 0.9583 & 0.9942 & 0.622 \\
\hline
\end{tabular}

Diffusion-based drug release can be either Fickian or non-Fickian [39]. In Fickian diffusion, the release rate is independent of the drug concentration in the dosage forms. The zero-order release kinetics model describes delivery systems where the drug release rate is constant with respect to time. In non-Fickian diffusion, several factors can affect the drug release, and based on these factors, the release can be predicted by several mathematical models like the first-order release kinetics model, the Higuchi release kinetics model, and the Korsmeyer-Peppas release kinetics model, etc. In first-order release kinetics, the drug release rate is concentration-dependent. The Korsmeyer-Peppas release kinetic model relates the drug release exponentially to the fractional release of the drug [40]. The equation can be written as follows:

$$
Q t / Q \infty=k t^{n}
$$

where $Q t / Q \infty$ is a fraction of drug released at time $t ; k$ is the release rate constant; and $n$ is the release exponent. 
The value of $n$ can indicate likely mechanisms for drug release. For example, Ritger and Peppas determined that $n$ should be in the range of $0.425-0.500$ for disk-shaped devices if the release profile indicates that diffusion of dissolved drug through a matrix is the dominant release mechanism [41,42]. The $n$ value of about 1.0 indicates that polymer relaxation, polymer dissolution, or tablet erosion is the dominant mechanism. An intermediate value suggests a combination of mechanisms is effective, involving the so-called non-Fickian diffusion, anomalous release, or mixed transport.

Among the shell compositions investigated, the release of propranolol $\mathrm{HCl}$ from shell A (containing $20 \%$ cellulose acetate and $65 \%$ mannitol) was best fitted to first-order kinetics, with an $\mathrm{R}^{2}$ value of 0.9939 , showing that the release rate depends on its concentration. The release of propranolol $\mathrm{HCl}$ from shells B and C (containing $40 \%$ and $60 \%$ cellulose acetate and $45 \%$ and $25 \%$ mannitol, respectively) was best fitted to the Korsmeyer-Peppas model, with $\mathrm{R}^{2}$ values of 0.9954 and 0.9959 , respectively. $n$-Values ranged between 0.576 and 0.619 , indicating a non-Fickian diffusion model of drug release.

Similarly, the release of propranolol $\mathrm{HCl}$ from the tablets enclosed in shells (the composition of formulation B), including fixed-, medium- and large-sized shells, was best fitted to the Korsmeyer-Peppas model, with $\mathrm{R}^{2}$ values of $0.9954,0.9964$, and 0.9942 , respectively. Shell sizes with fixed-, medium-, and large geometry showed $n$-values of $0.619,0.621$, and 0.622 , respectively. This indicates a non-Fickian diffusion model of drug release. Among the three geometries, the fixed size was more compact; therefore, less area was available for exposure to the dissolution media and hence the release rate was lower compared to drug release from the medium and the large shell. In contrast, the medium and large sizes of the shell provided more area for exposure to the dissolution media, leading to faster disintegration of the tablet and thus an improved release rate of propranolol $\mathrm{HCl}$.

The current approach shows that the modulation of the shell formulation and size represents an opportunity to control the amount and rate of propranolol $\mathrm{HCl}$ released from the off-the-shelf tablet. This novel idea can be developed further and applied to encapsulate the available conventional dosage forms to fulfil the needs of personalized medicine. This new perspective on the use of $3 \mathrm{D}$ printing could be applied to customize the amount and release profile of the enclosed available conventional drug tablet instead of developing a 3D printing model for each drug.

Promising dosage forms with modified-release profiles as per desirability have been fabricated using 3D printing technology [6]. However, the content verification, the formulation supply of each drug, and the expected competition between the conventional and the 3D printed dosage forms of the same drug are among the various difficulties found in the application of 3D printing in the healthcare system [8]. Here, we suggest that the encapsulation of conventional immediate-release tablets using 3D printed coating shells will bridge the gap between the currently available conventional tableting methods and the future applications of 3D printing technology to meet the final goal, which is to provide effective treatment with minimum adverse effects.

\section{Conclusions}

The fabrication of an encapsulating shell using extrusion-based 3D printing at room temperature showed no significant impact on the physicochemical character of the materials used to design the encapsulating system. This method provided the ability to use pharmaceutical grade excipients in different ratios without affecting the quality of the printed product. The use of different ratios of cellulose acetate to D-mannitol for the fabrication of the encapsulating shell showed the ability to control the amount and the rate of propranolol $\mathrm{HCl}$ released from off-the-shelf tablet (Indicardin ${ }^{\circledR}$ ). Further, the dissolution profile of the 3D printed controlled-release shell of different sizes also influenced the release of propranolol $\mathrm{HCl}$, in which the combination of material composition and shell size allowed for a fine-tuning of the release profile. This approach could be explored further to help healthcare professionals customize the drug amount and the release profile of the available conventional tablets to provide the greatest therapeutic efficacy with the minimum untoward effects commonly associated with the administration of the immediate-release tablets. 
Author Contributions: M.S.A.: Conceptualization, Methodology, Investigation, Writing—Review and Editing, Visualization, Supervision, Project Administration, and Funding Acquisition. A.A.M.: Methodology, Investigation, and Writing-Original Draft Preparation. J.A.: Methodology, Investigation, and Writing-Original Draft Preparation. E.S.: Software and Writing-Review and Editing. All authors have read and agreed to the published version of the manuscript.

Funding: This research was funded by Deanship of Scientific Research at Najran University under grant number NU/MID/16/068.

Acknowledgments: The authors are thankful to the Deanship of Scientific Research at Najran University for funding this work under research grant number NU/MID/16/068.

Conflicts of Interest: The authors declare no conflict of interest.

\section{References}

1. Adams, J.U. Pharmacogenomics and personalized medicine. Nat. Educ. 2008, 1, 194.

2. Tremblay, J.; Hamet, P. Role of genomics on the path to personalized medicine. Metabolism 2013, 62, S2-S5. [CrossRef]

3. Mathew, E.; Pitzanti, G.; Larrañeta, E.; Lamprou, D.A. 3D printing of pharmaceuticals and drug delivery devices. Pharmaceutics 2020, 12, 266. [CrossRef] [PubMed]

4. Khaled, S.A.; Burley, J.C.; Alexander, M.R.; Yang, J.; Roberts, C.J. 3D printing of five-in-one dose combination polypill with defined immediate and sustained release profiles. J. Control Release 2015, 217, 308-314. [CrossRef] [PubMed]

5. Robles-Martinez, P.; Xu, X.; Trenfield, S.J.; Awad, A.; Goyanes, A.; Telford, R.; Basit, A.W.; Gaisford, S. 3D printing of a multi-layered polypill containing six drugs using a novel stereolithographic method. Pharmaceutics 2019, 11, 274. [CrossRef]

6. Sun, Y.; Soh, S.L. Printing Drug Tablets with Fully Customizable Release Profiles for Personalized Medicine. U.S. Patent Application No. 15/742, 754, 2 August 2018.

7. Arafat, B.; Qinna, N.; Cieszynska, M.; Forbes, R.T.; Alhnan, M.A. Tailored on demand anti-coagulant dosing: An in vitro and in vivo evaluation of 3D printed purpose-designed oral dosage forms. Eur. J. Pharm. Biopharm. 2018, 128, 282-289. [CrossRef]

8. AlGahtani, M.S.; Mohammed, A.A.; Ahmad, J. Extrusion-based 3D printing for pharmaceuticals: Contemporary research and applications. Curr. Pharm. Des. 2019, 24, 4991-5008. [CrossRef]

9. Kyobula, M.; Adedeji, A.; Alexander, M.R.; Saleh, E.; Wildman, R.; Ashcroft, I.; Gellert, P.R.; Roberts, C.J. 3D inkjet printing of tablets exploiting bespoke complex geometries for controlled and tuneable drug release. J. Control Release 2017, 261, 207-215. [CrossRef]

10. Wang, J.; Goyanes, A.; Gaisford, S.; Basit, A.W. Stereolithographic (SLA) 3D printing of oral modified-release dosage forms. Int. J. Pharm. 2016, 503, 207-212. [CrossRef]

11. Fina, F.; Goyanes, A.; Gaisford, S.; Basit, A.W. Selective laser sintering (SLS) 3D printing of medicines. Int. J. Pharm. 2017, 529, 285-293. [CrossRef]

12. Goyanes, A.; Fina, F.; Martorana, A.; Sedough, D.; Gaisford, S.; Basit, A.W. Development of modified release 3D printed tablets (printlets) with pharmaceutical excipients using additive manufacturing. Int. J. Pharm. 2017, 527, 21-30. [CrossRef] [PubMed]

13. Li, Q.; Guan, X.; Cui, M.; Zhu, Z.; Chen, K.; Wen, H.; Jia, D.; Hou, J.; Xu, W.; Yang, X.; et al. Preparation and investigation of novel gastro-floating tablets with 3D extrusion-based printing. Int. J. Pharm. 2018, 535, 325-332. [CrossRef] [PubMed]

14. Yi, H.-G.; Choi, Y.-J.; Kang, K.S.; Hong, J.M.; Pati, R.G.; Park, M.N.; Shim, I.K.; Lee, C.M.; Kim, S.; Cho, D.-W. A 3D-printed local drug delivery patch for pancreatic cancer growth suppression. J. Control Release 2016, 238, 231-241. [CrossRef] [PubMed]

15. Holländer, J.; Genina, N.; Jukarainen, H.; Khajeheian, M.; Rosling, A.; Mäkilä, E.M.; Sandler, N. Three-dimensional printed PCL-based implantable prototypes of medical devices for controlled drug delivery. J. Pharm. Sci. 2016, 105, 2665-2676. [CrossRef]

16. Genina, N.; Holländer, J.; Jukarainen, H.; Mäkilä, E.M.; Salonen, J.J.; Sandler, N. Ethylene vinyl acetate (EVA) as a new drug carrier for 3D printed medical drug delivery devices. Eur. J. Pharm. Sci. 2016, 90, 53-63. [CrossRef] 
17. Stewart, S.A.; Domínguez-Robles, J.; McIlorum, V.J.; Mancuso, E.; Lamprou, D.; Donnelly, R.F.; Larrañeta, E. Development of a biodegradable subcutaneous implant for prolonged drug delivery using 3D printing. Pharmaceutics 2020, 12, 105. [CrossRef]

18. Dumpa, N.R.; Bandari, S.; Repka, M.A. Novel gastroretentive floating pulsatile drug delivery system produced via hot-melt extrusion and fused deposition modeling 3d printing. Pharmaceutics 2020, 12, 52. [CrossRef]

19. Gil, E.C.; Colarte, A.I.; Bataille, B.; Pedraz, J.L.; Rodríguez, F.; Heinämäki, J. Development and optimization of a novel sustained-release dextran tablet formulation for propranolol HCl. Int. J. Pharm. 2006, 317, 32-39. [CrossRef]

20. Kurcubic, I.; Cvijic, S.; Filipcev, B.; Ignjatovic, J.; Ibric, S.; Djuris, J. Development of propranolol HCl bilayer mucoadhesive buccal tablets supported by in silico physiologically-based modeling. React. Func. Polym. 2020, 151, 104587. [CrossRef]

21. Medicines and Healthcare products Regulatory Agency. Propranolol HCl tablet. In British Pharmacopoeia; Medicines and Healthcare Products Regulatory Agency: London, UK, 2020; Volume III, pp. 1182-11833.

22. United States Pharmacopoeial Convention. The United States Pharmacopeia 35; National Formulary 30; United States Pharmacopeial Convention Inc.: Rockville, MD, USA, 2012; Volume 3, p. 4465.

23. Mehta, M.R.; Machhaliya, R.Y.; Patel, C.N.; Daraji, H.M. Formulation and evaluation of sublingual tablet of candesartan cilexetil. Int. J. Pharm. Res. BioSci. 2014, 3, 900-925.

24. Khaled, S.A.; Alexander, M.R.; Wildman, R.; Wallace, M.J.; Sharpe, S.; Yoo, J.; Roberts, C.J. 3D extrusion printing of high drug loading immediate release paracetamol tablets. Int. J. Pharm. 2018, 538, 223-230. [CrossRef]

25. Junior, J.M.M.; Muller, A.L.H.; Foletto, E.L.; da Costa, A.B.; Bizzi, C.; Müller, E.I. Determination of propranolol hydrochloride in pharmaceutical preparations using near infrared spectrometry with fiber optic probe and multivariate calibration methods. J. Anal. Methods Chem. 2015, 2015, 795102. [CrossRef]

26. Ali, A.; Iqbal, M.; Akhtar, N.; Khan, H.M.S.; Ullah, A.; Uddin, M.; Khan, M.T. Assessment of xanthan gum based sustained release matrix tablets containing highly water-soluble propranolol HCl. Acta Pol. Pharm. 2013, 70, 283-289. [PubMed]

27. Sahoo, J.; Murthy, P.N.; Biswal, S.; Sahoo, S.K.; Mahapatra, A.K. Comparative study of propranolol hydrochloride release from matrix tablets with KollidonSR or hydroxy propyl methyl cellulose. AAPS PharmSciTech 2008, 9, 577-582. [CrossRef] [PubMed]

28. Khaled, S.A.; Burley, J.C.; Alexander, M.R.; Yang, J.; Roberts, C.J. 3D printing of tablets containing multiple drugs with defined release profiles. Int. J. Pharm. 2015, 494, 643-650. [CrossRef] [PubMed]

29. Khaled, S.A.; Burley, J.C.; Alexander, M.R.; Roberts, C.J. Desktop 3D printing of controlled release pharmaceutical bilayer tablets. Int. J. Pharm. 2014, 461, 105-111. [CrossRef]

30. Wan, S.; Sun, Y.; Qi, X.; Tan, F. Improved bioavailability of poorly water-soluble drug curcumin in cellulose acetate solid dispersion. AAPS PharmSciTech 2011, 13, 159-166. [CrossRef] [PubMed]

31. Patel, H.; Patel, M. Formulation and evaluation of controlled porosity osmotic drug delivery system of metoprolol succinate. Int. J. Pharm. Res. 2012, 3, 1761-1767.

32. Li, D.; Jiang, Y.; Lv, S.; Liu, X.; Gu, J.; Chen, Q.; Zhang, Y. Preparation of plasticized poly (lactic acid) and its influence on the properties of composite materials. PLOS ONE 2018, 13, e0193520. [CrossRef]

33. Laboulfie, F.; Hémati, M.; Lamure, A.; Diguet, S. Effect of the plasticizer on permeability, mechanical resistance and thermal behaviour of composite coating films. Powder Technol. 2013, 238, 14-19. [CrossRef]

34. Brobyn, R.D. The human toxicology of dimethyl sulphoxide. Ann. N. Y. Acad. Sci. 1975, 243, 497-506. [CrossRef] [PubMed]

35. Jacob, S.W.; Jack, C. Dimethyl Sulfoxide (DMSO) in Trauma and Disease; CRC Press: Boca Raton, FL, USA, 2015.

36. Dash, S.; Murthy, P.N.; Nath, L.; Chowdhury, P. Kinetic modeling of drug release from controlled drug delivery systems. Acta Pol. Pharm. 2010, 67, 217-223. [PubMed]

37. Kjar, A.; Huang, Y. Application of Micro-Scale 3D Printing in Pharmaceutics. Pharmaceutics 2019, 11, 390. [CrossRef]

38. Grassi, M.; Grassi, G. Mathematical modelling and controlled drug delivery: Matrix systems. Curr. Drug Deliv. 2005, 2, 97-116. [CrossRef] [PubMed]

39. Gokhale, A. Achieving zero-order release kinetics using multi-step diffusion-based drug delivery. Pharm. Tech. 2014, 26, 38-42. 
40. Korsmeyer, R.W.; Gurny, R.; Doelker, E.; Buri, P.; Peppas, N.A. Mechanisms of solute release from porous hydrophilic polymers. Int. J. Pharm. 1983, 15, 25-35. [CrossRef]

41. Ritger, P.L.; Peppas, N.A. A simple equation for description of solute release I. Fickian and non-fickian release from non-swellable devices in the form of slabs, spheres, cylinders or discs. J. Control Release 1987, 5, 23-36. [CrossRef]

42. Ritger, P.L.; Peppas, N.A. A simple equation for description of solute release II. Fickian and anomalous release from swellable devices. J. Control Release 1987, 5, 37-42. [CrossRef]

(C) 2020 by the authors. Licensee MDPI, Basel, Switzerland. This article is an open access article distributed under the terms and conditions of the Creative Commons Attribution (CC BY) license (http://creativecommons.org/licenses/by/4.0/). 